\title{
Parking on a Random Tree
}

\author{
H.G. Dehling • S.R. Fleurke • C. Külske
}

Received: 23 November 2007 / Accepted: 11 June 2008 / Published online: 16 July 2008

(c) The Author(s) 2008. This article is published with open access at Springerlink.com

\begin{abstract}
Consider an infinite tree with random degrees, i.i.d. over the sites, with a prescribed probability distribution with generating function $G(s)$. We consider the following variation of Rényi's parking problem, alternatively called blocking RSA (random sequential adsorption): at every vertex of the tree a particle (or "car") arrives with rate one. The particle sticks to the vertex whenever the vertex and all of its nearest neighbors are not occupied yet.

We provide an explicit expression for the so-called parking constant in terms of the generating function. That is, the occupation probability, averaged over dynamics and the probability distribution of the random trees converges in the large-time limit to $\left(1-\alpha^{2}\right) / 2$ with $\int_{\alpha}^{1} \frac{x d x}{G(x)}=1$.
\end{abstract}

Keywords Car parking problem $\cdot$ Random tree $\cdot$ Random sequential adsorption $\cdot$ Particle systems

\section{Introduction}

In the classical car parking problem considered by Rényi [10], one dimensional cars with unit length appear one by one with their midpoints uniformly distributed over an interval. A car is parked unless it intersects with one or more previously placed cars. The process

\footnotetext{
H.G. Dehling

Fakultät für Mathematik, Ruhr-Universität Bochum, Universitätsstraße 150, 44780 Bochum, Germany e-mail: herold.dehling@rub.de

url: http://www.ruhr-uni-bochum.de/ffm/Lehrstuehle/Lehrstuhl-XII/dehling.html

S.R. Fleurke

Agentschap Telecom, Postbus 450, 9700 AL Groningen, The Netherlands

e-mail: sjoert.fleurke@at-ez.nl

C. Külske (凶)

Department of Mathematics and Computing Sciences, University of Groningen, Nijenborg 9,

9747 AG Groningen, The Netherlands

e-mail: kuelske@math.rug.nl

url: http://www.math.rug.nl/ kuelske/
} 
stops, when there is no further possibility of placing a car. In the discrete version of Rényi's car parking problem, cars of length 2 try to park at their midpoints randomly on the integers. Now a car can be parked if the distance of its midpoint to all other midpoints of already parked cars is two or more. It is well known that the probability that a given site is occupied by the midpoint of a car converges to $\left(1-e^{-2}\right) / 2$ in the fully parked state, that is when time tends to infinity $[2,7]$. In higher dimensions a model of this type will in general not be solvable and more complicated behavior is expected to occur. In physical language, this process is called (blocking) RSA (random sequential adsorption), the motivation being that particles are deposited onto a surface [2, 5, 6, 9]. For a nice review article over various packing problems (of which the parking problem can also be seen as a special example), see [1].

In this note we consider the problem of parking on a random tree, where particles at the vertices appear with an exponential waiting time, and parking is prohibited when there is a car-midpoint already at the site or at a neighboring site on the tree. We stress that the tree itself is unchanged under the dynamics. We solve the model and calculate the occupation probability as a function of time for random trees with i.i.d. degrees, averaged over the distribution of trees. In particular we recover the regular tree result [9]. We believe that the random tree model has appeal for two reasons: The first is that it provides a step to the analysis of the process on random networks, motivated e.g. by the study of communication networks. Indeed, many of the random graph distributions proposed for its description which have found recent interest allow for a local random tree approximation [3, 4, 11]. The second reason why it is nice is just the mathematical simplicity of the result which should not go unnoticed.

The proof is based on an analysis of the ordinary differential equation of the occupation probability at a fixed site of a fixed realization of the random tree. This is like [7, 9], but we give a self-contained exposition. Conditioning on a non-arrival of a car at this site the r.h.s. of this differential equation allows for a factorization in terms of occupation probabilities on rooted trees. Again a differential equation for these quantities can be derived. While it cannot be expected to be solvable for a particular realization, the averaging over the tree distribution still allows for closed-form expressions, as we shall see.

\subsection{Random Trees with Independent Identically Distributed Degrees}

The precise definition of the model is as follows. We consider a random tree with vertices $i$ and degree at the site $i$ given by $D_{i}$. We choose $D_{i}$ to be independent random variables with the same distribution $\mathbb{Q}$ given by

$$
\mathbb{Q}\left(D_{i}=k\right)=a_{k}
$$

on the integers starting from 2. The latter requirement ensures that we have no open ends with probability one. We denote the generating function of the distribution by

$$
G(s)=\sum_{k=2}^{\infty} a_{k} s^{k}
$$

We will denote the expected value with respect to this probability distribution by the same symbol $\mathbb{Q}$. 


\subsection{The Dynamics}

Fix a tree with vertex set $V$. For any such tree we will define a Markov jump process on the occupation numbers $n=\left(n_{i}\right)_{i \in V} \in \Omega=\{0,1\}^{V}$. Here $n_{i}$ denotes the occupation number of vertex $i$, meaning that

$$
n_{i}= \begin{cases}0 & \text { if vertex } i \text { is vacant } \\ 1 & \text { if vertex } i \text { is occupied }\end{cases}
$$

The dynamics of the process is defined in terms of the generator which is given by the r.h.s. of the differential equation

$$
\frac{d}{d t} \mathbb{E}^{n} f(n(t))=\sum_{k}\left[f\left(n^{k}\right)-f(n)\right] r_{k}(n)
$$

with

$$
n_{i}^{k}= \begin{cases}n_{i} & \text { if } k \neq i \\ 1 & \text { if } k=i\end{cases}
$$

and

$$
\begin{aligned}
r_{k}(n) & =\prod_{i: d(i, k) \leq 1} n_{i}^{c}, \\
n_{i}^{c} & =1-n_{i},
\end{aligned}
$$

and where $d$ denotes the distance on the tree.

This generator defines a Markov jump process on the infinite graph by standard theory [8], such that (1.3) holds for any local function $f: \Omega \rightarrow \mathbb{R}$. Here $\mathbb{E}^{n}$ denotes the expected value with respect to the process, started at the initial configuration $n$.

This generator describes the parking of cars at all possible sites $k$ at rate 1 given a configuration which is given by $n$. This parking is possible whenever the site and its nearest neighbors are vacant, that is $r_{k}(n)=1$. Since at most one car can be parked at a given site, it suffices to consider the time of the first arrival of a car at this site, for all sites.

\subsection{Results}

We provide an explicit integral formula for the density of occupied sites at time $t$, averaged with respect to dynamics $\mathbb{E}$ and tree distribution $\mathbb{Q}$.

\section{Theorem 1}

$$
\mathbb{Q E}\left(n_{0}(t)\right)=\frac{1-\alpha^{2}\left(1-e^{-t}\right)}{2}
$$

where the function $\alpha(u)$ is defined by the equation

$$
\int_{\alpha(u)}^{1} \frac{x d x}{G(x)}=u
$$

In particular, the occupation probability, averaged over dynamics and the probability distribution of the random trees converges in the large-time limit to $\left(1-\alpha^{2}\right) / 2$ with $\int_{\alpha}^{1} \frac{x d x}{G(x)}=1$. 
Remark Let us specialize to the deterministic case of a regular tree with $D \geq 2$ nearest neighbors. We obtain

$$
\mathbb{E}\left(n_{0}(t)\right)= \begin{cases}\frac{1}{2}\left(1-e^{-2\left(1-e^{-t}\right)}\right) & \text { if } D=2 \\ \frac{1}{2}\left(1-\left(1+(D-2)\left(1-e^{-t}\right)\right)^{-\frac{2}{D-2}}\right) & \text { if } D>2\end{cases}
$$

and recover in this way the known results on the integers and general regular trees $[2,7,9]$.

\section{Proof}

Consider a fixed realization of the random tree and look at the time-evolution given by (1.3). We use a short notation for the probability that a given set of sites $A$ is empty at time $t$,

$$
C_{t}(A):=\mathbb{E}\left(\prod_{i \in A} n_{i}^{c}(t)\right)
$$

Similarly we write for the conditional probability that a given set of sites $A$ is empty, conditional on the event that on another set of sites $B$ no particles have arrived yet:

$$
C_{t}(A \mid B):=\mathbb{E}\left(\prod_{i \in A} n_{i}^{c}(n) \mid T_{j}>t \quad \text { for } j \in B\right)
$$

Of course, if no particle has arrived at a site it is empty, but the converse is not true. Here $T_{j}$ denotes the arrival time of the particle at site $j$. The $T_{j}$ 's are i.i.d. exponentially distributed with expected value equal to one. We also write in short $C_{t}\left(i_{1}, \ldots, i_{k}\right) \equiv C_{t}\left(\left\{i_{1}, \ldots, i_{k}\right\}\right)$ for $A=\left\{i_{1}, \ldots, i_{k}\right\}$ etc.

As the underlying tree is random, also these correlation functions are random variables with respect to the distribution $\mathbb{Q}$. For any realization we can write the dynamics (1.3) as

$$
-\frac{d}{d t} C_{t}(A)=C_{t}(\bar{A})
$$

where $\bar{A}=\{i \in G \mid d(i, A) \leq 1\}$. Similarly we have for the dynamics of the conditional correlation function the differential equation

$$
\begin{aligned}
-\frac{d}{d t} C_{t}(A \mid B) & =C_{t}(\bar{A} \mid B) \\
& =C_{t}\left(\bar{A} \cap B^{c} \mid B\right)
\end{aligned}
$$

The first equation follows from restricting the generator to the set $B^{c}$ (since in $B$ no particles have arrived yet). The second equation expresses the fact that when no particles have arrived in the set $B$, it implies that $\bar{A} \cap B$ is unoccupied.

Fix an arbitrary vertex. Let us call this vertex 0 . Starting from the dynamics for the correlation functions (2.3) we have

$$
\begin{aligned}
-\frac{d}{d t} C_{t}(0) & =C_{t}(\{i, d(i, 0) \leq 1\}) \\
& =\mathbb{E}\left(\prod_{i, d(i, 0) \leq 1} n_{i}^{c}(t) 1_{T_{0}>t}\right)+\mathbb{E}\left(\prod_{i, d(i, 0) \leq 1} n_{i}^{c}(t) 1_{T_{0} \leq t}\right)
\end{aligned}
$$


We notice that the second term vanishes since an arrival of a particle at 0 would imply that the sites $\{i, d(i, 0) \leq 1\}$ cannot all be empty. Writing the first term as a conditional probability we get

$$
-\frac{d}{d t} C_{t}(0)=C_{t}(\{i, d(i, 0) \leq 1\} \mid 0) e^{-t}
$$

Conditional on the event that no particle has arrived at the site 0 , the time-evolutions for the occupation numbers on the branches of the tree that are emerging from 0 are independent. Consequently the correlations factorize into a product and we have

$$
C_{t}(\{i, d(i, 0) \leq 1\} \mid 0)=\prod_{i, d(i, 0)=1} C_{t}(i \mid 0)
$$

where the $C_{t}(i \mid 0)$ is the non-occupation probability of a site $i$ that is adjacent to the root on a rooted tree, assuming that no particle has arrived at the root.

For a particular realization of the underlying tree, these functions will in general differ. Let us however now take the expected value over the tree distribution $\mathbb{Q}$. We can decompose this average into an average over $D_{0}$ (the number of neighbors of the central vertex 0 ) and a conditional average over the remaining branches, rooted at 0 . Conditioning the number of branches emerging from zero to be equal to $k$, the correlation functions are independent random variables with respect to $\mathbb{Q}$ and so we obtain

$$
\mathbb{Q}\left(C_{t}(\{i, d(i, 0)=1\} \mid 0) \mid D_{0}=k\right)=\left(\mathbb{Q}\left(C_{t}(1 \mid 0)\right)\right)^{k}
$$

where we denoted by the site 1 one of the nearest neighbors of 0 , see Fig. 1.

From here, (2.6) and (2.7) we have

$$
\begin{aligned}
-\frac{d}{d t} \mathbb{Q}\left(C_{t}(0)\right) & =\sum_{k=2}^{\infty} a_{k}\left(\mathbb{Q} C_{t}(1 \mid 0)\right)^{k} e^{-t} \\
& =G\left(\mathbb{Q} C_{t}(1 \mid 0)\right) e^{-t}
\end{aligned}
$$

where the first line follows by dominated convergence. Integrating this equation gives us the desired function $\mathbb{Q}\left(C_{t}(0)\right)$ once the "half-infinite function" $\mathbb{Q} C_{t}(1 \mid 0)$ is known. So, it remains to solve a problem on a rooted tree, conditioned on the event that no particle has arrived at the root, averaged over $\mathbb{Q}$.

Fig. 1 Fragment of a tree where sites 0 and 1 are empty and the occupancy of the other sites is unknown

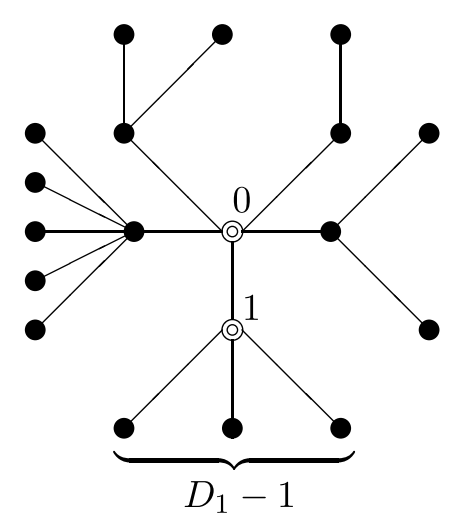


Indeed, it turns out to be possible to derive a closed-form differential equation for this object, as we show now. Note at first that, as in (2.5) ff., it suffices to consider the event that no particle has arrived at 1 and write

$$
C_{t}(\{i, d(i, 1) \leq 1\} \mid 0)=C_{t}(\{i, d(i, 1) \leq 1\} \mid 0,1) e^{-t}
$$

Since the root 0 and the site 1 itself are empty under the conditioning we have

$$
\begin{aligned}
C_{t}(\{i, d(i, 1) \leq 1\} \mid 0,1) & =C_{t}(\{i \neq 0, d(i, 1)=1\} \mid 0,1) \\
& =\prod_{i \neq 0, d(i, 1)=1} C_{t}(i \mid 0,1)
\end{aligned}
$$

Employing the dynamics (2.4), using (2.10) and averaging over $\mathbb{Q}$ we thus have

$$
\begin{aligned}
-\frac{d}{d t} \mathbb{Q} C_{t}(1 \mid 0) & =\mathbb{Q} C_{t}(\{i, d(i, 1) \leq 1\} \mid 0) \\
& =\mathbb{Q}\left(\prod_{i \neq 0, d(i, 1)=1} C_{t}(i \mid 0,1)\right) e^{-t}
\end{aligned}
$$

Using conditional independence over the branches with respect to the tree distribution $\mathbb{Q}$ and noting that $\mathbb{Q} C_{t}(i \mid 0,1)=\mathbb{Q} C_{t}(1 \mid 0)$ for the $i$ 's appearing in the above product to get

$$
\mathbb{Q}\left(\prod_{i \neq 0, d(i, 1)=1} C_{t}(i \mid 0,1)\right)=\sum_{k=2}^{\infty} a_{k}\left(\mathbb{Q} C_{t}(1 \mid 0)\right)^{k-1}
$$

we finally obtain with (2.12) and (2.13) the closed-form differential equation

$$
-\frac{d}{d t} \mathbb{Q} C_{t}(1 \mid 0)=\frac{G\left(\mathbb{Q} C_{t}(1 \mid 0)\right)}{\mathbb{Q} C_{t}(1 \mid 0)} e^{-t}
$$

Its solution $\mathbb{Q} C_{t}(1 \mid 0)$ with initial condition $\mathbb{Q} C_{t=0}(1 \mid 0)=1$ is given by the integral

$$
\int_{\mathbb{Q} C_{t}(1 \mid 0)}^{1} \frac{y d y}{G(y)}=1-e^{-t}
$$

Let us substitute this solution into the equation for the filling probability (2.9). This then implies the explicit formula for the $\mathbb{Q}$-average of the filling density of a random tree with generating function $G$ of the form

$$
\mathbb{Q E}\left(n_{0}(t)\right)=\int_{0}^{t} G(y(s)) e^{-s} d s
$$

where $y(s)$ is defined by the equation

$$
\int_{y(s)}^{1} \frac{x d x}{G(x)}=1-e^{-s}
$$


Finally, defining the function $s(u)$ by $u=1-e^{-s(u)}$ we have $\int_{y(s(u))}^{1} \frac{x d x}{G(x)}=u$ which implies $\frac{d y(s(u))}{d u}=-\frac{y(s(u))}{G(y(s(u)))}$ and recalling (1.6) we see that

$$
\begin{aligned}
\int_{0}^{t} G(y(s)) e^{-s} d s & =\int_{0}^{1-e^{-t}} G(y(s(u))) d u=-\int_{1}^{\alpha\left(1-e^{-t}\right)} y d y \\
& =\frac{1}{2}\left(1-\alpha^{2}\left(1-e^{-t}\right)\right)
\end{aligned}
$$

This finishes the proof.

Open Access This article is distributed under the terms of the Creative Commons Attribution Noncommercial License which permits any noncommercial use, distribution, and reproduction in any medium, provided the original author(s) and source are credited.

\section{References}

1. Chaikin, P.: Random thoughts. Phys. Today 60, 8-9 (2007)

2. Cohen, R., Reiss, H.: Kinetics of reactant isolation I. One-dimensional problems. J. Chem. Phys. 38(3), 680-691 (1963)

3. Dorogovtsev, S.N., Mendes, J.F.F.: Evolution of Networks. From Biological Nets to the Internet and WWW. Oxford University Press, Oxford (2003)

4. Durrett, R.: Random Graph Dynamics. Cambridge Series in Statistical and Probabilistic Mathematics. Cambridge University Press, Cambridge (2007)

5. Evans, J.W.: Random and cooperative sequential adsorption. Rev. Mod. Phys. 64(4), 1281-1327 (1993)

6. Gouet, R., Sudbury, A.: Blocking and dimer processes on the Cayley tree. J. Stat. Phys. doi:10.1007/s10955-007-9451-5

7. Hemmer, P.C.: The random parking problem. J. Stat. Phys. 57, 865-869 (1989)

8. Liggett, T.M.: Interacting Particle Systems. Springer, New York (1985)

9. Penrose, M.D., Sudbury, A.: Exact and approximate results for deposition and annihilation processes on graphs. Ann. Appl. Prob. 15(1B), 853-889 (2005)

10. Rényi, A.: On a one-dimensional problem concerning random space-filling. Publ. Math. Inst. Hung. Acad. Sci. 3, 109-127 (1958)

11. van der Hofstad, R., Hooghiemstra, G., Znamenski, D.: Distances in random graphs with finite mean and infinite variance degrees. Electron. J. Probab. 12(25), 703-766 (2007) 